\title{
Application of Knowledge of Fluid Mechanics in the Field of Design of Forming Tools
}

\author{
Ján Moravec ${ }^{1, *}$ \\ ${ }^{1}$ University of Žilina, Faculty of Mechanical Engineering, Department of Technological Engineering, \\ Univerzitná 8215/1, 01026 Žilina, Slovak Republic
}

\begin{abstract}
The paper deals with the construction of a hydrostatic molding tool. The theoretical part presents the necessary knowledge about forming in liquid media. The experimental part presents the construction of a forming tool for drawing using a liquid. The discussion deals with environmental constraints of production processes.
\end{abstract}

\section{Introduction}

During 100 years long history of metal forming was beside usage of conventional methods also used methods unconventional. Unconventional methods will have, with regard to environmental restrictions, bigger importance than in the past.

One method of non-conventional forming is the forming in liquid environment forming by applying hydrostatic pressure in the forming tool (FT).

\section{Theoretical part}

Liquid pressure on a planar surface element of fixed wall is proportional to the size of the surface element according to the specific pressure of the fluid in the immediate vicinity of the surface element. The size of the total pressure is according to the following statement: considering the size of the flat surface $S$, forming an angle $\alpha$ with the horizontal plane. The surface $S$ is in the horizontal direction and in the direction of maximum slope area divided into elementary sections, thus creating elementary rectangles (one with a shaded area $d S$ ) - Figure 1 .

Pressure $P$ on the surface $S$ is equal to the limit toward which the sum of the elementary pressure $d P$ on the surface elements $d S$, the dimensions of these elements tend to zero:

$$
P=\iint_{S} d P .
$$

Let the symbol $z$ be the depth of the mass centre of the marked surface element. Expression of $d P$ and $P, d P=\gamma \cdot z \cdot d S$ :

\footnotetext{
*Corresponding author: jan.moravec@,fstroj.uniza.sk
} 


$$
P=\iint_{S} \gamma \cdot z \cdot d S=\gamma \cdot \iint_{S} z \cdot d S
$$

Let the symbol $y$ be the distance of centre of pressure $d P$ in the marked area element from the intersection of the $x$ plane surface with a horizontal plane $\Delta 0$ :

$$
P=\gamma \cdot \iint_{S} y \cdot \sin \alpha \cdot d S=\gamma \cdot \sin \alpha \cdot \iint_{S} y \cdot d S
$$

where $\iint_{S} y \cdot d S$ is the static moment of area with respect to the $x$ axis. If $T$ is a mass centre of area $S$ and $y T$ its distance from $x$, by definition, the sum of the moments of components is equal to the resultant torque on the axis:

$$
y_{T}=\frac{\iint_{S} y \cdot d S}{S},
$$

and $P=\gamma \cdot \sin \alpha \cdot y_{T} \cdot S$. Because $y_{T} \cdot \sin \alpha=z_{T}$, where $z_{T}$ is the depth of mass centre surface $S$, will ultimately be:

$$
P=\gamma \cdot S \cdot z_{T}
$$

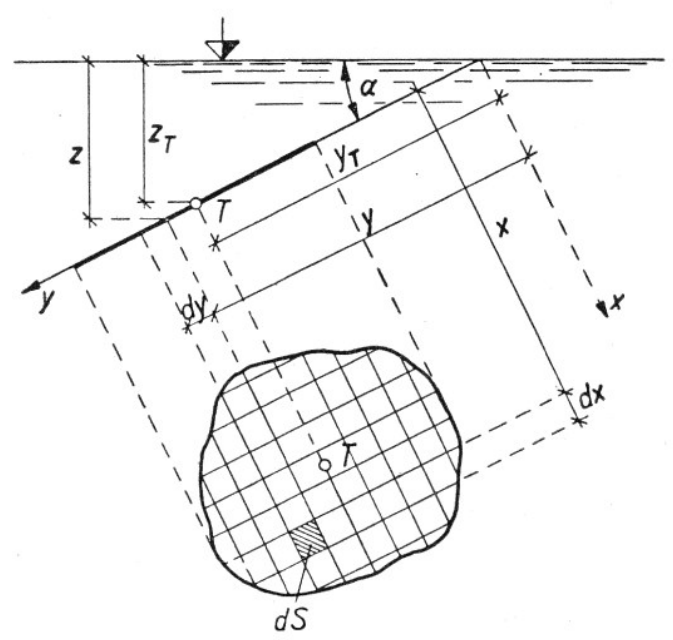

Fig. 1. Calculation of the total pressure on the flat surface of any shape.

With an increase of pressure on the liquid, volume is reduced by a very small value. When the pressure is reduced to its original value, the liquid immediately take its original volume. Liquids are therefore perfectly elastic. Compressibility of the fluid is evaluated by a coefficient of compressibility $(K S)$, which is defined by (2):

$$
\gamma=-\frac{1}{V} \cdot \frac{d V}{d p}
$$

where $V$ is the initial liquid volume, $d V$ is the elemental reduction $V$ caused by elementary increase of pressure about value $d p$. $K S$ varies for different liquids. At high pressures is decreasing, with increasing temperature generally increases. $K S$ for water at $20^{\circ} \mathrm{C}$ is: $\gamma=$ 4.998. $10^{-10}\left(\mathrm{~Pa}^{-1}\right)$. 
In the case of forming in the liquid environment is in the process applied also physics knowledge, known as Pascal's Law.

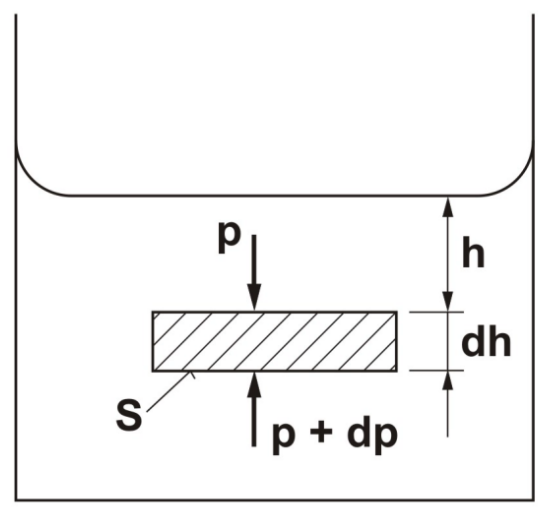

Fig. 2. Derivation of equations.

Equilibrium conditions: the vector sum of all forces $=0$. Weight of selected quantity of liquid has the value:

$$
d G=\rho \cdot S \cdot g \cdot d h
$$

and the size of the resulting pressure forces in the vertical direction oriented upward (buoyancy):

$$
d f=(p+d p) \cdot S-p \cdot S=S \cdot d h .
$$

From the equilibrium conditions is resulting following equation: $\rho \cdot S \cdot g \cdot d h-S \cdot d p=0$ (3). After editing and integrating equation (1): $p-\rho \cdot g \cdot h=$ const. (4).

Potential of the earth's gravitational field at a depth $h$ below the surface of the liquid may be expressed by relation, if the liquid surface potential $=0: V=-g \cdot h$, and equation (4) goes to the form: $p+\rho \cdot V=$ const. (5). Equation (5) is of general scope: when the incompressible fluid is at calm state in any force field, the sum of the potential energy of its volumetric element and pressure in the total volume of liquid equal. If the liquid is under more pressure and does not have too much volume (potential changes in the liquid are small), $\rho \cdot V$ in equation (6) can be considered constant and from it can be concluded this relationship: $p=$ const. (7), which is a statement of Pascal's Law integrally spreading pressure in liquids [1-5].

\section{Application}

In case of sheet molding are preferably processed materials based on metal blanks. Also for this reason is forming in the hydraulic environment of great perspective. In process of forming with $F T$ in liquid environment, this liquid can replace one active element of $F T$. One of the factor is therefore also reduction of the economic costs of their production. Liquid environment forming, although it is not entirely new method, is still somehow, despite its advantages, in the shade of the whole-metal FT. This fact is surprising because this method can eliminate various negative impacts of production processes, because it uses water as the active element, the element that does not pollute the environment. 


\subsection{Experiments}

The aim of experiments was to design and produce $F T$ that, thanks to application of proper knowledge and patterns, will allow to form metal parts. The intention was to implement the dragging tools. Principle of hydrostatic forming is based on effect of hydrostatic pressure $p H$ in FT and pressing tool. Among the methods used for dragging sheet are very important methods using for this purpose the elastic - plastic environment. In this case is used so called non-fixed tool. Compared to fixed tools it has a number of benefits - lower production costs for $F T$, especially for smaller batches of complex shapes products. Furthermore, the simplification of the forming process by the elimination of necessarily to fit both of the FT active parts together and also it is not necessary to use complex retainers. Company Cincinati Milling Machine Co. developed in 1951 (!) method for deep drawing with help of liquid environment - Hydroform. In this method, the universal dragging tool includes container with water or oil, which is sealed with a rubber membrane. Fixed dragging part is made of a cheap steel. Production costs of the whole dragging tool represent only $25-30 \%$ of the classic all-metal construction $F T$.

In the following text, attention is focused to the issue of application of this method in sheet metal manufacturing, with use of special FT for laboratory conditions.

\subsection{Principle}

According to Figure 3 FT consists of two parts - the bottom and top. Position (1) and (7) are machined rotary lightweight Al castings. Their shape was chosen because of ease of machining such an area.

To the bottom (vessel) is injected through hydraulic system fluid, which develops pressure on the piston (position 2) into which are screwed three guiding pins (position 4) with the dragging part (position 5). The container is closed by a top cover (position 3). In the upper part is placed a circular cross member (position 6), in which are drilled holes for guide pins (position 4). Also dragging part is located there (position 5). Cavity of holder (position 7) is filled with polyurethane, which allows forming of circular trim from deepdrawed sheet metal. For the reason that these work represent experimental verification method, the shape of product was very simple. In Figure 3 and 4 the method of $F T$ attachment to pressing machine is shown. Verification of experiment has showed that $F T$ was designed correctly and it is possible to deform simple shapes. Processed parts are placed on the crosspiece and accurate rails with aid of hinges (item 4) will allow easy operation.

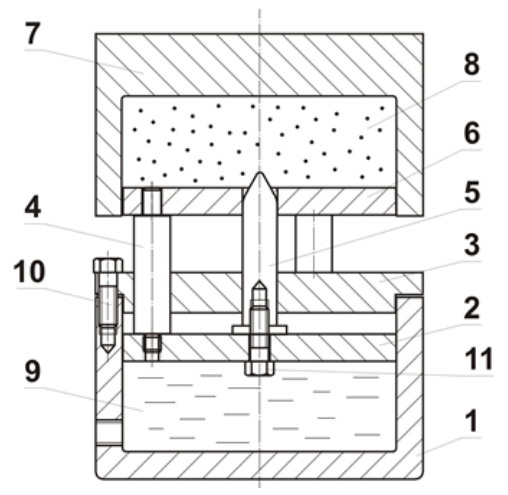

Fig. 3. Scheme of the used tool: 1 - wessel, 2 - piston, 3 - cover, 4 - pins, 5 - drager, 6 - cros member, 7 - holder, 8 - rubber, 9 - pressure liquid, 10 - screw M10, 11 - screw M10x20 mm. 

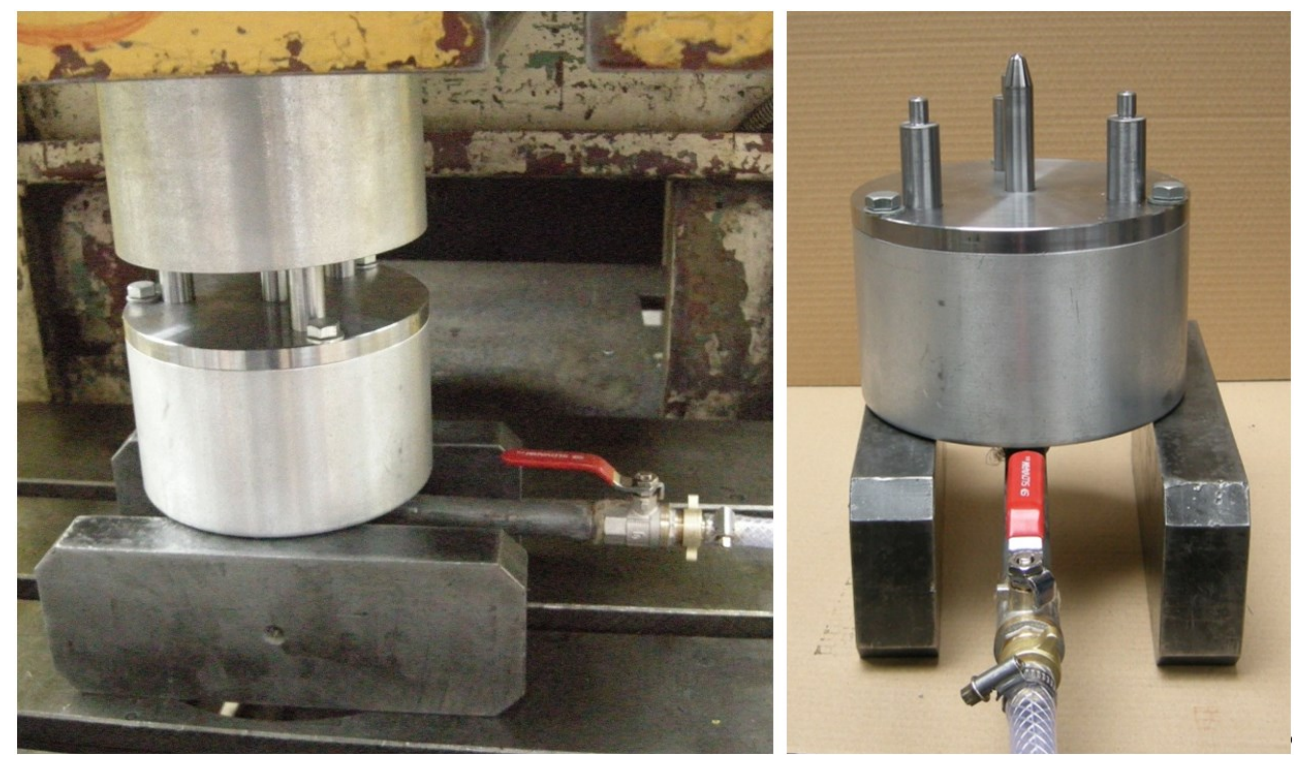

Fig. 4. Tool attached on press (left), bottom part of the tool (right).

\section{Discussion}

Physical knowledge from the field of fluid mechanics are a great source of information. Applying the (generally) any findings in this sector - reflected in the technical solution almost always contains one restriction - the impact of used solutions on the environment. During recent years, this condition becomes the limiting factor of either technical or technological applications. It is essential and necessary to respect the fact that the earth consists of one huge ecosystem regardless of national boundaries. In the sector of mechanical engineering will be imperative to give priority to organic solutions. That is the reason why our experiments were focused on the proposed contribution. Use of water as a basic element of living nature in the production process is one of the roads leading to this goal [6-8].

Overall assessment of the experimental work:

- FT is implemented differently from the scheme in Figure 3. Inlet is not located on the side panel, but the pressure fluid is fed through the bottom.

- Tests were mainly focused on verifying the functionality $F T$.

- Number of experimental samples was small (five pieces) and therefore cannot be on those findings, formulate general conclusions.

Experiments can be assessed positively, because the findings confirmed the correctness of the structure proposed and implemented, thus the main objective was fully achieved.

\section{Conclusion}

The presented contribution was focused on unconventional forming of metal parts. The biggest advantage is the achievement of hydro dragging coefficient $m=0.3$ in one operation, in which yet another methods failed. A smaller amount of operations reduces the price $F T$, which will eventually (for certain amount of products) reduce price up to ten times. Connected to this also possibility to use hydro dragging method even for smaller productions. The exact shape of the final product has only dragger and median formed by 
pressure fluid contributes to reducing the overall cost of the FT production. The unquestionable advantages of hydro-mechanical dragging are:

- Financial savings when dragging conical and parabolic parts.

- Sheet in the process becomes thinner only 2-3\%, thus maintaining the same thickness of sheet throughout the process.

- Rippling caused by dragging causes greater forming possibilities, thereby improving the mechanical properties of the material.

The disadvantage is the increase in tensile strength, resulting in the need to use more powerful pressing machines [9-11].

Presented FT is a suitable aid for teaching forming technologies. Experimental studies have confirmed the appropriateness of this type of forming especially for piece production and application tool extends the sphere of physical knowledge usable in sheet metal forming.

\section{References}

1. J. Moravec, Hydroforming (EDIS - University of Zilina, 2016)

2. S. Kundar, Technology of Metal Forming Processes (London Press, 2004)

3. V. Boljanovic, Sheet Metal Forming processes and the Design (London, 2005)

4. R. Pearce, Sheet Metal forming (Vienna, 2010)

5. Schuler, GmbH, Metal Forming Handbook (Springer Verlag Berlin, 2008)

6. W. F. Hosford, R. M. Caddel, Metal forming, Mechanics and Metallurgy (Cambridge, 2007)

7. Z. Marciniak, J. L. Duince, S. J. Hu, Sheet Metal Forming (New York, 2004)

8. P. Durcansky, S. Papucik, J. Jandacka, M. Holubcik, R. Nosek, SCI WORLD J., 138254 (2014)

9. M. Holubčík, N. Kantová, J. Jandačka, Z. Kolková, MATEC Web Conf, 168, 06005 (2018)

10. M. Vasko, M. Blatnicky, P. Kopas, M. Saga, Metalurgija 56 (2017)

11. S. Steisunas, J. Dizo, G. Bureika, V. Zuraulis, Procedia Engineering 187 (2017) 